\title{
Early oral contraceptive use and breast cancer: theoretical effects of latency
}

\author{
KLIM M c PHERSON, P A COOPE*, AND M P VESSEY \\ From the Department of Community Medicine and General Practice, Radcliffe Infirmary, Oxford OX2 $6 H E$
}

SUMMARY Many cancers and other chronic diseases are associated with a long delay between exposure to a putative risk factor and subsequent diagnosis. This presents well recognised problems in the elucidation of suspected risk factors by epidemiological methods. In this paper we discuss the interpretation in epidemiological studies of the effect of a possible risk factor when population exposure is recent and rapidly changing. An important contemporary example concerns the study of early oral contraceptive $(O C)$ use in relation to the subsequent risk of breast cancer. Computer simulations reported here indicate that plausible delays in the manifestation of any effect on breast cancer incidence make it difficult to exclude early $\mathrm{OC}$ use as a risk factor for breast cancer, even when large well conducted epidemiological studies show no apparent increased risk. Methods for detecting a 'latent' effect are discussed.

The recent report in the Lancet $^{1}$ of a large case control study of early oral contraceptive (OC) use (ie, use at a young age or before first term pregnancy) by the Cancer and Steroid Hormone (CASH) Group in the USA showed no statistically significant increase in breast cancer incidence under the age of 45 years associated with such OC exposure. A number of other studies, however, have shown some effect. ${ }^{24}$ The editorial ${ }^{5}$ which accompanied the Lancet publication cautioned against the drawing of final conclusions from the CASH evidence on the grounds that epidemiological studies have shown significant associations of, for example, exposure to ionising radiation $^{6}$ and stilboestrol ${ }^{7}$ during pregnancy, with breast cancer, but sometimes not until 15 to 20 years after exposure. The editorial suggested that since early OC use is a relatively recent phenomenon (and apparently more so in the USA than in the UK) it might be too early to see any effect. It was claimed that latency could be an important cause of bias in case control studies, that is to say that the inevitable inclusion as controls of women who were either in a preclinical phase, or for whom the carcinogenic process had not ended, or for whom some final stage in a multi-stage process had not yet happened, would bias the estimates of relative risk downwards.

What we call in this paper a 'latent effect' is possibly the sum of an induction period during which cell changes are taking place which eventually lead to

* Present address: Christchurch, New Zealand cancer, as well as a true latent period in which the disease is present but not detected. Rothman ${ }^{8}$ calls the whole period the empirical induction period. The true latent period has to do with the detection of a tumour in the breast, and clearly this tends to happen more commonly once the tumour has reached a palpable size than when it is very small. It is argued by some', from considerations of cell kinetics, that this delay might sometimes be as long as ten years. Much less, of course, is known about the induction period because in the case of breast cancer it is not even clear what the causative agent is. The carcinogenic process could be continuous but relatively slow or there could be several discrete stages ${ }^{10}$ between each of which there is a characteristic time distribution. For instance some stage could be a random mutation of an already transformed cell line. Such a mutation, if an entirely random one, could lead to an exponential distribution of time between stages. On the other hand, exposure to some other agent which might be required to accomplish a transformation at any stage might be far from random and strongly age dependent or dependent on menstrual or obstetric events. Then the time between stages could have a more Gaussian or log Gaussian distribution.

Clearly, while the existence of some kind of latent effect is plausible, its exact nature is difficult to discern. Cigarette smoking is known to be a cause of lung cancer, and the sex specific incidence and mortality of lung cancer appear to relate to patterns of use after a delay of around 20 years. Moreover, a case 
control study by Doll and Hill in $1950^{11}$ yielded a relative risk of lung cancer with smoking among men of 14 , but among women the relative risk was only $2 \cdot 5$. Clearly this difference could have been attributable to age at starting to smoke or method of smoking, but men began to smoke in the UK at a much earlier date than women. ${ }^{12}$ However the relationship between cigarette smoking and lung cancer is different from the problem considered here because, first, the causative relationship is now almost unquestioned; secondly, duration of exposure is much longer; thirdly, smoking causes most of the disease: and, fourthly, it is likely that cigarettes are important in both early and late stage carcinogenesis.

Breast cancer is relatively common with or without OCs and its relationship with early $O C$ use remains thoroughly questionable. As we have indicated, some studies show a significant association and others do not. Stadel and his co-workers have emphasised bias in recall and the selection of controls as a possible explanation for the results of the positive studies. ${ }^{1}$ While this clearly remains plausible, it is difficult to prove. We investigate here the existence and magnitude of another possible source of bias*-that of latency, when combined with recent and rapidly changing exposure patterns.

\section{Method}

THE SIMULATION MODEL

We used a computer program written by one of us (PAC) to simulate the exposure to OCs and subsequent diagnosis of breast cancer in hypothetical cohorts of women 'born' in each calendar year from 1930 to 1965 . To estimate the prevalence of exposure to OCs we analysed the data from 2246 controls included in case control studies of breast cancer conducted in this department between 1968 and 1984 . These controls are women who matched within five years of age with breast cancer cases and who were being treated in hospital; some were also matched by parity. A description of control selection is described in detail elsewhere. ${ }^{13} \mathrm{We}$ derived the proportions of these women who had been exposed to OCs before first term pregnancy by year of birth. In the simulation, each woman generated was assigned randomly, according to those proportions, to one of three groups describing her exposure to OCs: never used, up to four years' use, four or more years' use.

Incorporated into the simulation were the England and Wales age-specific risks of breast cancer, ${ }^{14}$ so that each woman generated had the appropriate agespecific incidence applied to her. The incidence for a woman exposed to OCs was multiplied by a factor

*We use the word 'bias' in its most generic sense, i.e., estimated relative risks which turn out to be incorrect, in the sense that they are incomplete. some time (the latent interval) after her exposure. We chose two hypothetical associations of early OC exposure with breast cancer incidence. The first assigned a relative risk of 1.4 to 'up to four years' OC use' and 3.0 to 'four or more years' OC use', and the second double these risks, $2 \cdot 8$ and 6.0 respectively.

The latent interval determined at which point after OC exposure the incidence of breast cancer was multiplied by this relative risk. Data on mean age at first OC use by year of birth were obtained by interpolation and extension of figures from $O C$ use before first term pregnancy among our controls. ${ }^{4} 1315$ For any woman, age at first OC used was determined randomly from a Gaussian distribution with the appropriate mean, and a standard deviation of four years, subject to the constraint that this age could not be less than 15 years, and such that OCs could not have been used before the year 1962. The time at which the latent interval began was arbitrarily taken as three years after the start of OC use for women with 0-4 years' exposure, and five years after for those with four or more years' exposure.

We considered five separate patterns of latency, of which the first was no latent interval. The remaining four patterns consisted of a variable latent interval with a Gaussian distribution and,

mean 5 years, standard deviation 2 years, mean 10 years, standard deviation 4 years, mean 15 years, standard deviation 4 years, mean 20 years, standard deviation 4 years.

We chose a Gaussian distribution because latency could be the sum of several independent random time delay processes such as a subclinical period, a prolonged carcinogenic process or the sum of the times between successive stages and the final stage in a multi stage process. We chose the stated standard deviations so that at least for some average latencies there was effectively a minimum latent period. The evidence already cited does suggest that no excess cancers in an exposed group need be observed for the first ten or 15 years. We also considered an exponential and a log normal distribution ${ }^{16}$ with similar mean durations.

ANALYSIS OF SIMULATED DATA

Cases of breast cancer were generated by this method, and for every case, a woman of the same age (and obviously completely comparable in respect of all risk factors except for exposure to OCs) was randomly selected as a control. Such pairs were then accumulated over quinquennia of calendar time and analysed as matched case control studies. The analysis initially ignored the period between exposure and diagnosis, as is commonplace in the reporting of the results of case control studies. This process was repeated for successive quinquennia from 1975-79 to 1995-99. The size of the annual cohorts of simulated 
births was chosen so that the number of case control pairs in each quinquennium would be sufficient for the estimate of relative risk to be precise enough to detect even quite small biases. We then analysed the simulated data, taking account of a possible latency, to recapture the imposed pattern.

\section{Results}

Although analyses were done at two levels of exposure, we will mainly report the results of estimated relative risks associated with four or more years' use of OCs before first pregnancy. This is because the results concerning lesser periods of exposure are predictable, given these other results. Table 1 shows the pattern of exposure experienced by our controls interviewed between 1968 and 1983.

It can be seen that exposure is uncommon among women born before 1934, and long-term exposure is uncommon among women born before 1944. In these tabulations any OC use among nulliparous women is taken as use before first term pregnancy. In our view, use of OCs before first term pregnancy can be regarded as providing an indication of OC use before age 25 , because clearly these are correlated. Henceforth we refer to such use as 'early use'. It should be noted that early use patterns of women born after 1950 are likely to be unstable because in our sample the numbers of women are small. We assumed that for women born after 1959 (ie, until 1965) the early use patterns were the same as for those born between 1955 and 1959. This is probably conservative because OC use among the young increased during the seventies.

After several trial runs we settled on cohorts of 50000 women 'born' in each calendar year between 1930 and 1965. This yielded enough cases in each quinquennium to minimise the effects of chance on relative risk estimates, and provided sufficiently narrow confidence intervals on these estimates to be secure in estimating the effects of bias. The analysis of

Table 1 Oral contraceptive (OC) use in nulliparous women, or before first term pregnancy in parous women (\%)

\begin{tabular}{lccccccc}
\hline & \multicolumn{6}{c}{ Year of birth } \\
\cline { 2 - 8 } OC use & $\begin{array}{l}\text { Before } \\
1929\end{array}$ & $1930-34$ & $1935-39$ & $1940-44$ & $1945-49$ & $1950-54$ & $1955-59$ \\
\hline Never & 99 & 97 & 94 & 86 & 59 & 43 & 27 \\
$<4$ yr & 1 & 3 & 5 & 12 & 29 & 43 & 27 \\
$4+\mathrm{yr}$ & 0 & 0 & 1 & 2 & 12 & 14 & 45 \\
$\begin{array}{l}\text { No. of } \\
\text { women }\end{array}$ & 621 & 646 & 498 & 268 & 154 & 48 & 11 \\
\hline
\end{tabular}

such a sample size took several hours to run on an IBM AT micro computer.

Since the proportion of women exposed for four or more years among women diagnosed, for instance, in 1980 is only non-zero among the under 45s, and among those diagnosed in 1985 among the under $50 \mathrm{~s}$, the corresponding case control studies included only such women as were eligible. Thus, in the last quinquennium, women up to the age of 65 were included. This means that the number of case control pairs in early periods is much smaller than in later periods because breast cancer becomes increasingly common as age increases. Broadly, however, there were approximately 2000 pairs in the first period and 10000 in the last. A birth cohort of 50000 is approximately one-fifth of the actual female birth rate in England and Wales per annum, and these figures should reflect roughly one-fifth of the incident cases up to the ages specified in appropriate five-year intervals. The results are summarised in table 2 assuming that the true relative risk associated with $4+$ years of early $\mathrm{OC}$ use is 3 . The $95 \%$ confidence intervals on the estimates of relative risk are also given.

Because the biases entirely attributable to plausible latent periods are large, an obvious implication is that even positive studies may underestimate the ultimate relative risk. We repeated the simulations with double the 'true' relative risk to see if even these hypothetical estimates could be consistent with currently observed results if there happened to be a particularly long latent period. These results are shown in table 3. An exponential or log Gaussian distribution of latency with the same mean values as we have used and similar

Table 2 Estimated relative risk (with 95\% confidence limits) of breast cancer after 4 or more years of early $O C$ use by calendar period and latency with 'true' relative risk of 3

\section{Calendar Period}

Latency (yr)

( \pm standard deviation)

$\begin{array}{lllll}1975-79 \quad 1980-84 & 1985-89 & 1990-94 & 1995-99\end{array}$

\begin{tabular}{llllll}
\hline None & $3 \cdot 1$ & $3 \cdot 1$ & $3 \cdot 2$ & $2 \cdot 9$ & $3 \cdot 0$ \\
& $(2 \cdot 2,4 \cdot 4)$ & $(2 \cdot 5,3 \cdot 8)$ & $(2.8,3 \cdot 7)$ & $(2 \cdot 6,3 \cdot 2)$ & $(2 \cdot 8 \cdot 3 \cdot 3)$ \\
$5 \pm 2$ & $2 \cdot 4$ & $2 \cdot 6$ & $2 \cdot 7$ & $3 \cdot 0$ & $3 \cdot 0$ \\
& $(1 \cdot 6,3 \cdot 6)$ & $(2 \cdot 1,3 \cdot 3)$ & $(2 \cdot 3,3 \cdot 1)$ & $(2 \cdot 7,3 \cdot 4)$ & $(2 \cdot 7,3 \cdot 2)$ \\
$10 \pm 4$ & & & & & \\
& $1 \cdot 6$ & $1 \cdot 4$ & $2 \cdot 1$ & $2 \cdot 8$ & $3 \cdot 0$ \\
$15 \pm 4$ & $(1 \cdot 0,2 \cdot 4)$ & $(1 \cdot 1,1 \cdot 8)$ & $(1 \cdot 8,2 \cdot 4)$ & $(2 \cdot 5,3 \cdot 2)$ & $(2 \cdot 7,3 \cdot 2)$ \\
& $1 \cdot 2$ & $1 \cdot 6$ & $1 \cdot 8$ & $2 \cdot 1$ & 2.6 \\
$20 \pm 4$ & $(0.7,1 \cdot 8)$ & $(1.2,2 \cdot 0)$ & $(1 \cdot 6,2 \cdot 2)$ & $(1 \cdot 9,2 \cdot 4)$ & $(2 \cdot 4,2 \cdot 9)$ \\
& & & & & \\
& $1 \cdot 0$ & $1 \cdot 0$ & $1 \cdot 1$ & $1 \cdot 6$ & $2 \cdot 0$ \\
& $(0 \cdot 6,1 \cdot 5)$ & $(0 \cdot 8,1 \cdot 4)$ & $(0 \cdot 9,1 \cdot 4)$ & $(1 \cdot 4,1 \cdot 8)$ & $(1 \cdot 8,2 \cdot 2)$
\end{tabular}

Age of cases

and controls

$\leq 45$

$\leq 50$

$\leq 55$

$\leq 60$

$\leq 65$ 
Table 3 Estimated relative risk (with 95\% confidence limits) of breast cancer after 4 or more years of early $O C$ use by calendar period and latency with 'true' relative risk of 6

\begin{tabular}{|c|c|c|c|c|c|}
\hline \multirow[b]{2}{*}{$\begin{array}{l}\text { Latency ( } y r) \\
( \pm \text { standard deviation) }\end{array}$} & \multicolumn{5}{|c|}{ Calendar Period } \\
\hline & $1975-79$ & $1980-84$ & $1985-89$ & $1990-94$ & $1995-99$ \\
\hline None & $\begin{array}{l}5 \cdot 7 \\
(4 \cdot 2,7 \cdot 7)\end{array}$ & $\begin{array}{l}6 \cdot 0 \\
(5 \cdot 0,7 \cdot 1)\end{array}$ & $\begin{array}{l}6 \cdot 6 \\
(5 \cdot 9,7 \cdot 4)\end{array}$ & $\begin{array}{l}6 \cdot 6 \\
(6 \cdot 0,7 \cdot 2)\end{array}$ & $\begin{array}{l}6 \cdot 7 \\
(6 \cdot 3,7 \cdot 2)\end{array}$ \\
\hline $5 \pm 2$ & $\begin{array}{l}4 \cdot 3 \\
(3 \cdot 1,6 \cdot 0)\end{array}$ & $\begin{array}{l}5 \cdot 5 \\
(4 \cdot 6,6 \cdot 7)\end{array}$ & $\begin{array}{l}5 \cdot 3 \\
(4 \cdot 7,6 \cdot 0)\end{array}$ & $\begin{array}{l}6 \cdot 4 \\
(5 \cdot 8,6 \cdot 9)\end{array}$ & $\begin{array}{l}6 \cdot 7 \\
(6 \cdot 3,7 \cdot 2)\end{array}$ \\
\hline $10 \pm 4$ & $\begin{array}{l}2 \cdot 0 \\
(1 \cdot 4,3 \cdot 0)\end{array}$ & $\begin{array}{l}2 \cdot 7 \\
(2 \cdot 2,3 \cdot 4)\end{array}$ & $\begin{array}{l}4 \cdot 0 \\
(3 \cdot 5,4 \cdot 6)\end{array}$ & $\begin{array}{l}5 \cdot 7 \\
(5 \cdot 2,6 \cdot 3)\end{array}$ & $\begin{array}{l}6 \cdot 2 \\
(5 \cdot 8,6 \cdot 7)\end{array}$ \\
\hline $15 \pm 4$ & $\begin{array}{l}1 \cdot 2 \\
(0 \cdot 8,2 \cdot 0)\end{array}$ & $\begin{array}{l}2 \cdot 1 \\
(1 \cdot 6,2 \cdot 7)\end{array}$ & $\begin{array}{l}2 \cdot 7 \\
(2 \cdot 3,3 \cdot 1)\end{array}$ & $\begin{array}{l}4 \cdot 1 \\
(3 \cdot 7,4 \cdot 6)\end{array}$ & $\begin{array}{l}5 \cdot 0 \\
(4 \cdot 6,5 \cdot 3)\end{array}$ \\
\hline $20 \pm 4$ & $\begin{array}{l}1 \cdot 3 \\
(0 \cdot 8,2 \cdot 0)\end{array}$ & $\begin{array}{l}1 \cdot 4 \\
(1 \cdot 1,1 \cdot 8)\end{array}$ & $\begin{array}{l}1 \cdot 6 \\
(1 \cdot 4,1 \cdot 9)\end{array}$ & $\begin{array}{l}2 \cdot 3 \\
(2 \cdot 1,2 \cdot 6)\end{array}$ & $\begin{array}{l}3 \cdot 5 \\
(3 \cdot 2,3 \cdot 7)\end{array}$ \\
\hline $\begin{array}{l}\text { Age of cases } \\
\text { and controls }\end{array}$ & $\leq 45$ & $\leq 50$ & $\leq 55$ & $\leq 60$ & $\leq 65$ \\
\hline
\end{tabular}

standard deviations made little difference to the extent or time pattern of the biases.

As already indicated, it can be seen that plausible latent periods can be associated with serious bias in case control studies. Indeed, if the latent period is more than ten years on average, even large case control studies will underestimate the ultimate risk until the end of the century if relatively young women are included in the analyses. Table 4 illustrates the bias in the relative risk estimates for different ages, pooled over calendar period. Of course, the biases disappear when an analysis includes only cases and controls for all of whom the latent period has expired. As can be seen from the table, this typically means over the age of 45 for an average latency of 10 years, 50 for a latency of 15 years, and 55 for 20 years' latency. Such investigations will not be possible for another 10-20 years.

Table 4 Estimated relative risk of breast cancer after $4+$ years of early $O C$ use, pooled over calendar period with 'true' relative risk of 3

\begin{tabular}{lllllllllll}
\hline \multicolumn{1}{l}{ Age $(y r)$} \\
\cline { 2 - 9 } Latency $(y r)$ & $20-24$ & $25-29$ & $30-34$ & $35-39$ & $40-44$ & $45-49$ & $50-54$ & $55-59$ & $60-64$ \\
\hline None & 2.1 & 2.3 & 2.7 & 3.3 & 2.9 & 3.4 & 2.9 & 2.9 & 2.7 \\
$5 \pm 2$ & 2.1 & $1.8^{*}$ & 2.2 & 3.2 & 3.0 & 2.8 & 3.2 & 2.9 & 4.2 \\
$10 \pm 4$ & 1.2 & $0.9^{*}$ & $1.9^{*}$ & $2.3^{*}$ & 2.7 & 2.9 & 2.9 & 3.7 & 3.4 \\
$15 \pm 4$ & 1.0 & $1.6^{*}$ & $1.3^{*}$ & $1.8^{*}$ & 2.3 & $2.6^{*}$ & 2.7 & 2.7 & 3.4 \\
$20 \pm 4$ & $0.6^{*}$ & $1.0^{*}$ & $1.0^{*}$ & $1.3^{*}$ & $1.4^{*}$ & $2.0^{*}$ & $2.0^{*}$ & 3.1 & 2.2 \\
\hline
\end{tabular}

* Significant difference $p<0.05$ from relative risk of 3
We estimated for the first pattern of relative risks $(1.4$ and 3.0) the proportion of cases of breast cancer attributable to early $\mathrm{OC}$ use assuming these relative risks and the exposure patterns of table 1 . These attributable risks depend crucially on the accuracy of the estimates of the proportions exposed and are derived by combining the results of a simulation run for which the relative risks were unity with those of the runs described above. Thus, estimates of attributable risks for later periods must be viewed with caution because the proportions of exposed women born after 1950 are based on small numbers (see table 1). Table 5 gives the estimated percentage increase in incidence attributable to early $O C$ use. Since the sampling distribution of these estimates is fairly wide they should not be taken as exact. However, it can be seen that for long latent periods noticeable changes in incidence cannot be expected before the end of this decade even if the relative risk for long term early exposure is around 3.0.

Finally, using the simulation program, we generated samples of cases and controls and analysed these simulated case control studies incorporating a possible latent effect into the analysis. Restricting the generation of simulated cases and controls to those 'collected' before 1985, the latency pattern imposed becomes clear, with the correct analysis. ${ }^{8}$ For instance, with a latency of 15 years, a standard deviation of four years and a relative risk of 3.0 , the usual analysis, as table 2 indicates, yields an estimated relative risk of around $1 \cdot 5$. If, however, all early OC use within eight years of diagnosis (or an equivalent date for the controls) is excluded from the analysis, the estimated relative risk for four or more years' early use starts to become a little larger. Successive exclusion of use within 10,12 , etc. years of diagnosis yields increasing estimates of relative risk. When all use within 15 years is excluded, the estimated relative risk is maximised at

Table 5 Estimated percentage increase in incidence of breast cancer attributable to early OC use, by latency and calendar period (assuming that the relative risk up to 4 years $O C$ use is 1.4 and 4 or more years is 3.0 )

\begin{tabular}{llllll}
\hline \multicolumn{5}{l}{ Calendar Period } \\
\cline { 2 - 6 } Latency (yr) & $1975-79$ & $1980-84$ & $1985-89$ & 1990.94 & 199599 \\
\hline None & 12 & 10 & 19 & 18 & 31 \\
$5 \pm 2$ & 10 & 11 & 16 & 20 & 32 \\
$10 \pm 4$ & 5 & 4 & 10 & 12 & 30 \\
$15 \pm 4$ & 2 & 2 & 7 & 8 & 21 \\
$20 \pm 4$ & 0 & 0 & 1 & 5 & 18 \\
\hline Age group & $\leq 45$ & $\leq 50$ & $\leq 55$ & $\leq 60$ & $\leq 65$ \\
\hline
\end{tabular}


nearly 3.0 -but this estimate has wide confidence limits because accumulating four years of early $O C$ use 15 years or more before 1985 was rare. In this example, the maximum dispersion of latent period would be estimated by $15-8$, ie., 7 , and if it has a symmetric Gaussian distribution this might approximate two standard deviations. Thus we might crudely estimate the standard deviation of the latent period as three and a half years with a mean of 15 years. Clearly, this is consistent with the latent period imposed by the model.

\section{Discussion}

It is clear that latency can be a cause of bias quite as important as other well-known sources of bias in case control studies such as recall, control selection, and confounding. In the absence of concrete knowledge about the real distribution of latency, it is often difficult to know precisely how to allow for it in analysis. This is particularly so when one considers that in real case control studies of the effects of OCs the time between starting OC use and diagnosis, duration of use, and age at starting $O C$ use are correlated. Moreover, the OC composition and dose is confounded with calendar period of use and therefore the period between OC use and diagnosis. Since all these factors could, in principle, be differentially associated with breast cancer, it is hardly surprising that there is uncertainty in the interpretation of existing studies. In addition to this there is probably important confounding between OC use and age at menarche, age at first term birth, benign breast disease, and a family history of breast cancer. Pike ${ }^{17}$ is right, therefore, to require us all to think harder about the true biological process.

In the analysis of current case control studies some indication of the presence of a bias attributable to latency, however, can be derived by successive calculations of relative risk excluding recent exposure among both cases and controls. For example, when early OC use within, say, eight years of diagnosis (and an equivalent date for the controls) is excluded from the exposure variable, and this results in an increase in relative risk, this is suggestive of a latent effect. If in turn the relative risk is increased by exclusion of use within ten, then 12 and more years of diagnosis, some estimate of the length and dispersion of the latency can be derived. The mean latency is then the period of OC use exclusion at which the relative risk estimate is first maximised, and the dispersion can be estimated from the point at which the estimated relative risk begins to rise. If, on the other hand, the relative risk shows no particular pattern with increasing exclusion of recent exposure, then latency would be unlikely to be a serious cause of bias, unless its length was well outside the maximum period between diagnosis and accumulated early OC use.

The available data $^{5}$ on pill use in the United Kingdom and the United States suggest that early pill use happened commonly in the United States later than in this country: around five years later seems a reasonable estimate. This is approximately equivalent to estimating the bias in case control studies in England and Wales for 1975-79 as if they applied in the United States in 1980-1984 (ie, five years later). Thus, we can see that the results of the CASH study could be consistent with a true relative risk of 3.0 if, for instance, the average latent period is more than ten years.

Moreover, if a latent period exists it might not be surprising that a case control study done before $1980,{ }^{13}$ which we have reported, yielded no association while another study done after $1980^{4}$ yielded a relative risk of nearly 3.0 . These two studies were done in essentially the same hospitals with the same interviewers, using almost the same protocol. The opportunity for different bias to have arisen from recall or control selection was therefore minimal.

In conclusion, the analysis of case control studies of chronic disease associations does call for explicit investigation of a latent effect. Such an effect is not implausible and, if corroborated by such analyses, may provide insight into biological mechanisms. In the example discussed in this paper, for instance, if such an effect were to be demonstrated, it would be likely that OCs acted as a promoter in early stage carcinogenesis.

\section{References}

${ }^{1}$ Stadel BV, Rubin GL, Webster L, et al. Oral contraceptives and breast cancer in young women. Lancet 1985; ii: 970-3.

2 Royal College of General Practitioners. Breast cancer and oral contraceptives in the RCGP study. Br Med J 1981; 282: 2089-93.

${ }^{3}$ Pike MC, Hederson BE, Krailo MD, et al. Breast cancer in young women and use of oral contraceptives. Possible modifying effect of formulation and age at use. Lancet 1983; ii: 970-3.

${ }^{4}$ McPherson K, Neil A, Vessey MP, Doll R. Oral contraceptives and breast cancer. Lancet 1983; ii: 1414.

5 Editorial. Another look at the pill and breast cancer. Lancet 1985; ii: 985-7.

6 Tokunaga M, Norman JE, Asano M. Malignant breast tumours among atomic bomb survivors, Hiroshima and Nagasaki. J Nat Cancer Inst 1979; 62: 1347-59.

${ }^{7}$ Greenberg ER, Barnes AB, Resseguie L, et al. Breast cancer in mothers given diethylstibestrol in pregnancy. New Engl Med 1984; 311: 1393-8.

8 Rothman KJ. Induction and latent periods. $\mathrm{Am} \mathrm{J}$ Epidemiol 1981; 114: 235-9.

${ }^{9}$ Buchanan JB, Spratt JS, Heuser LS. Tumour growth, doubling times and inability of the radiologist to diagnose certain cancers. Radio Clin $N$ Am 1983: 21: 115-26. 
${ }^{10}$ Day $\mathrm{N}$ E. Epidemiological data and multi-stage carcinogenesis. IARC Sci Publ 1984; 56:

${ }^{11}$ Doll R, Bradford Hill A. Smoking and carcinoma of the lung. $\mathrm{Br}$ Med $J$ 1950; ii: 739-48.

${ }^{12}$ Royal College of Physicians. Smoking or health. 1977, Pitman Medical.

${ }^{13}$ Vessey MP, Baron J, Doll R, McPherson K, Yeates D. Oral contraceptive and breast cancer. Final report on epidemiological study. Br J Cancer 1983; 47: 455-62.
${ }^{14}$ OPCS. OPCS Monitor MBI 85/2. Cancer Registration 1982.

${ }^{15}$ Vessey MP, McPherson K., Yeates D, Doll R. Oral contraceptive use and abortion before first term pregnancy in relation to breast cancer risk. $\mathrm{Br} J$ Cancer 1982; 45: 327-33.

${ }^{16}$ Armenian HK, Lilienfeld AM. Incubation periods of disease. Epidemiol Rev 1983; 5: 1-15.

${ }^{17}$ Pike MC. Breast cancer and oral contraceptives. Lancet 1985; ii: $1180-1$. 